\title{
Random conjugates of bankruptcy rules
}

\author{
Marieke Quant • Peter Borm
}

Received: 14 July 2009 / Accepted: 24 June 2010 / Published online: 9 July 2010

(C) The Author(s) 2010. This article is published with open access at Springerlink.com

\begin{abstract}
This article introduces and analyzes random conjugates of bankruptcy rules. A random conjugate is a rule which is derived from the definition of the underlying rule for two-claimant problems. For example, the random conjugate of the Aumann-Maschler rule yields an extension of concede-and-divide: the basic solution for bankruptcy problems with two claimants. Using the concept of random conjugates an alternative characterization of the proportional rule is provided. It turns out that the procedural definition of a random conjugate extends several of the properties of the underlying rule for two-claimant problems to the general domain of problems with an arbitrary number of claimants.
\end{abstract}

\section{Introduction}

Bankruptcy problems are first treated within the framework of interactive allocation problems in O'Neill (1982). In a bankruptcy problem a certain amount of money, the estate, has to be divided among a group of claimants. The amount claimed is larger than the estate, which gives rise to the problem of determining a fair division of the estate, satisfactory to all claimants. Many (bankruptcy) rules to solve these problems have been proposed in the literature. An overview of rules and their properties can be found in Thomson (2003).

Thomson and Yeh (2008) study the topic of "operators" on the space of all rules. In particular their interest is in the duality operator, the claims truncation operator, and

M. Quant $(\varangle) \cdot$ P. Borm

CentER and Department of Econometrics \& OR, Tilburg University, P.O. Box 90153, 5000 LE Tilburg,

The Netherlands

e-mail: quant@uvt.nl 
the minimal rights operator. Their study also includes the inheritance of properties of the original rule by the rule obtained from applying the operator.

In this article we introduce an operator that is in the same spirit as Thomson and Yeh (2008). In particular we consider random conjugates of rules which are derived from the definition of the underlying rule for two-claimant problems. Random conjugates are general rules, i.e., they are applicable to the domain of all problems. The random conjugate of a rule can thus be seen as the result of an "extension" operator that is applied to two-claimant rules. Formally, the extension operator differs from the operators defined in Thomson and Yeh (2008), since the domain of these operators is the space of all rules and not only two-claimant rules.

Two-claimant rules haven been extended to general rules in several ways. For example Thomson (2007) deals with the construction of a consistent extension (in the sense of Aumann and Maschler (1985)) whenever it exists, whereas Moulin (2000), Dominguez and Thomson (2006), Hokari and Thomson (2008), and Thomson (2008) consider consistent extensions of specific two-claimant rules. Furthermore, Dagan and Volij (1997) introduce a way to define an extension of any two-claimant rule that is so-called average consistent. Dagan and Volij (1997) and Hokari and Thomson (2008) answer the question which properties are preserved by the extension operator under consideration.

Random conjugates are the result of a different type of extension operator. It recursively uses the procedural definition of the original rule for two-claimant problems. The idea of a random conjugate is inspired by the extension of the standard solution of two-person cooperative games to a solution of cooperative games (the consensus value) with an arbitrary finite set of players provided by Ju et al. (2004). The underlying idea is that claimants leave the group one by one in a specific (but random) order. At the moment a claimant leaves the (remaining) group, he receives a part of the estate that is left at that stage. The amount he gets is based on the underlying rule for a two-claimant problem in which he is seen as one claimant and the rest of the group together as the other. In this way the underlying rule is applied recursively to a two-claimant setting. Taking the average over all possible orders, one then obtains the random conjugate of a rule.

The application of the random conjugate of a given rule can be motivated as follows. Suppose there is a bank that manages the estate, using a specific rule as its leading allocation principle. The bank knows the total amount of the claims, but is ignorant about the exact number of claimants. Now the claimants arrive in a specific (but random) order at the bank. So at the time a specific claimant arrives, the bank only knows the remaining estate, the claim of the present claimant, and the total claims of the possible claimants that are to arrive in future. Therefore, at that moment the bank applies its allocation principle in a two-claimant setting. After all claimants have arrived, an instance of the associated random conjugate will be the chosen allocation. The random conjugate averages over all instances corresponding to all possible orders.

Our first result will be an alternative characterization of the proportional rule, based on the procedural definition of a random conjugate. Moreover, we will show that the random conjugate of a rule is appealing in the sense that if some important properties (like invariance under claims truncation, self-duality, and minimal rights first) are 
satisfied by the rule in two-claimant problems, then these properties are inherited on the domain of all problems by the random conjugate.

Finally we note that the concept of random conjugates provides a way to extend the principles of rules that are only applicable to two-claimant problems to arbitrary problems. As an example one can think of concede-and-divide. In a problem with only two claimants, e.g., the run-to-the-bank rule (cf. O'Neill (1982)), the AumannMaschler rule (cf. Aumann and Maschler (1985)), and the adjusted proportional rule (cf. Curiel et al. (1988)) coincide. Thomson (2003) refers to this basic or standard rule for a two-claimant setting as concede-and-divide. The related concede-and-divide principle is also known as the contested garment principle (Aumann and Maschler (1985)). The idea behind concede-and-divide is appealing, and deserves an extension to problems with more than two claimants. Aumann and Maschler (1985) hint at a possible extension, but they only consider a fixed order (based on increasing claims) and restrict to intermediate sizes of the estate. Besides this extension mentioned in Aumann and Maschler (1985), the concede-and-divide principle has not been extended conceptually to problems with more than two claimants. The random conjugate of concede-and-divide, the so-called random concede-and-divide rule, does provide such a conceptual extension to arbitrary problems. In particular, by randomizing over all possible orders, it eliminates the arbitrariness of proceeding in the order of increasing claims as proposed by Aumann and Maschler (1985). A comprehensive analysis of the random concede-and-divide rule can be found in Quant et al. (2005). This article extends the ideas provided in Quant et al. (2005) to the more general framework of random conjugates associated to arbitrary rules.

This article is organized as follows: in Sect. 2 the formal definition of the random conjugate of a rule is introduced. A recursive formula is provided, and a new characterization of the proportional rule is derived. In Sect. 3 it is shown that the random conjugate of a rule extends important properties of the underlying rule in two-claimant problems to the domain of all problems.

\section{Random conjugates of rules}

This section formally introduces for each rule a random conjugate that is based on its definition for problems in which there are only two claimants present. In this way a new rule is created that is not only applicable to two-claimant problems, but to arbitrary problems.

A bankruptcy problem consists of a triple $(N, E, c)$, where $E$ is the estate that has to be divided among a finite set of claimants $N$, and $c \in \mathbb{R}^{N}, c \geq 0$ is a vector of claims. By the nature of a bankruptcy problem we assume that $E \leq \sum_{i \in N} c_{i}$. The class of all such problems is denoted by $\mathcal{C}$. Let $(N, E, c) \in \mathcal{C}$. A function $f$ on $\mathcal{C}$ is a (bankruptcy) rule if for all problems $(N, E, c)$ :

(i) $f(N, E, c) \in \mathbb{R}^{N}$,

(ii) $0 \leq f_{i}(N, E, c) \leq c_{i}$ for all $i \in N$,

(iii) $\sum_{i \in N} f_{i}(N, E, c)=E$. 
Moreover, we assume a rule $f$ to be anonymous, which basically means that the identity of an agent does not matter. An overview of rules proposed in the literature is found in Thomson (2003).

The random conjugate of a rule is based on the definition of the underlying rule in two-claimant problems and will respect the principles of this rule in its procedural definition. The underlying idea behind this random conjugate is that claimants leave the group one by one in a specific (but random) order. If a claimant leaves the group he receives a part of the estate. The amount given to him is based on the outcome given by the underlying rule for problems with two claimants. He himself is seen as one claimant, while the remaining group as a whole is viewed as the other claimant and where its claim is determined by adding all individual claims. The part that is distributed to the rest of the group acts as the new estate in the following step, when the next claimant is leaving. Taking the average over all possible orders results in the random conjugate of a rule.

An order of $N$ is a bijective function $\sigma:\{1, \ldots,|N|\} \rightarrow N$. The claimant at position $k$ in the order $\sigma$ is denoted by $\sigma(k)$. The set of all orders of $N$ is denoted by $\Pi(N)$. Let $f$ be a rule, $(N, E, c) \in \mathcal{C}$ and $\sigma \in \Pi(N)$. The order $\sigma$ is interpreted as the order in which claimants are leaving with a certain amount of the estate based on the rule $f$. This results in a vector $s^{\sigma, f}(N, E, c)$. Formally the vector $s^{\sigma, f}(N, E, c) \in \mathbb{R}^{N}$ is recursively defined as follows. Let $k \in\{1, \ldots,|N|\}$. Then

$$
s_{\sigma(k)}^{\sigma, f}(N, E, c)=f_{\sigma(k)}\left(\left\{\sigma(k), \sigma(k)^{c}\right\}, E^{k},\left(c_{\sigma(k)}, \sum_{\ell=k+1}^{|N|} c_{\sigma(\ell)}\right)\right)
$$

where $\sigma(k)^{c}$ is a claimant that can be seen as the combination of the claimants $\sigma(k+$ $1), \ldots, \sigma(|N|)$. His claim is the result of adding all the claims of these claimants. Furthermore, $E^{k}$ is the amount of the estate that is left if $\sigma(k)$ is leaving the group, so $E^{1}=E, E^{k}=E^{k-1}-s_{\sigma(k-1)}^{\sigma, f}(N, E, c)(2 \leq k \leq|N|){ }^{1}$

The random conjugate of a rule averages between all possible orders.

Definition Let $f$ be a rule. The random conjugate of $f$, denoted by $R f$, is defined by

$$
R f_{i}(N, E, c)=\frac{1}{|N| !} \sum_{\sigma \in \Pi(N)} s_{i}^{\sigma, f}(N, E, c)
$$

for all $i \in N$ and all $(N, E, c) \in \mathcal{C}$.

Note that in the case $|N|=2$, with $\sigma^{1}=(12)$ and $\sigma^{2}=(21), s^{\sigma^{1}, f}(N, E, c)=$ $s^{\sigma^{2}, f}(N, E, c)=f(N, E, c)$, for any rule $f$, estate $E$ and claim vector $c \in \mathbb{R}^{N}$. Hence, for two-claimant problems $(N, E, c), R f(N, E, c)=f(N, E, c)$.

\footnotetext{
${ }^{1}$ Note that $E^{k}$ depends on the rule $f$ under consideration. For expositional purposes, however, we omit $f$ from the notation.
} 
An alternative characterization of the random conjugate of a rule is provided in the following theorem.

Theorem 2.1 Let $(N, E, c) \in \mathcal{C}$. Then for all $i \in N$

$$
\begin{aligned}
R f_{i}(N, E, c)= & \frac{1}{|N|} f_{i}\left(\left\{i, i^{c}\right\}, E,\left(c_{i}, \sum_{j \in N \backslash\{i\}} c_{j}\right)\right) \\
& +\frac{1}{|N|} \sum_{j \in N \backslash\{i\}} R f_{i}\left(N \backslash\{j\}, E_{-j}, c_{-j}\right),
\end{aligned}
$$

where $i^{c}$ is a claimant that can be seen as a combination of all other claimants, $E_{-j}$ is the amount that is left of the estate if claimant $j$ is the first one to leave, i.e.,

$$
E_{-j}=E-f_{j}\left(\left\{j, j^{c}\right\}, E,\left(c_{j}, \sum_{k \in N \backslash\{j\}} c_{k}\right)\right)
$$

and $c_{-j} \in \mathbb{R}^{N \backslash\{j\}}$ denotes the claim vector $c$, in which the claim of claimant $j$ is omitted.

Proof Let $i \in N$. We analyze the payoff $R f_{i}(N, E, c)$ by considering the payoff of claimant $i$ in all possible orders. Let $\sigma \in \Pi(N)$.

Case 1: $\sigma(1)=i$. Claimant $i$ achieves

$$
s_{\sigma(1)}^{\sigma, f}=f_{i}\left(\left\{i, i^{c}\right\}, E,\left(c_{i}, \sum_{j \in N \backslash\{i\}} c_{j}\right)\right) .
$$

Case 2: $\sigma(1)=j$ and $j \neq i$. Let $\bar{\sigma} \in \Pi(N \backslash\{j\})$ be such that $\bar{\sigma}(k)=\sigma(k+1)$, $k \in\{1, \ldots,|N|-1\}$. Then

$$
s_{i}^{\sigma, f}(N, E, c)=s_{i}^{\bar{\sigma}, f}\left(N \backslash\{j\}, E_{-j}, c_{-j}\right),
$$

which directly follows from the recursive definition of the vector $s^{\sigma, f}(N, E, c)$.

Hence,

$$
\begin{aligned}
& R f_{i}(N, E, c)=\frac{1}{|N| !} \sum_{\sigma \in \Pi(N)} s_{i}^{\sigma, f}(N, E, c) \\
& =\frac{1}{|N| !} \sum_{\substack{\sigma \in \Pi(N): \\
\sigma(1)=i}} s_{i}^{\sigma, f}(N, E, c)+\frac{1}{|N| !} \sum_{j \in N \backslash\{i\}} \sum_{\substack{\sigma \in \Pi(N): \\
\sigma(1)=j}} s_{i}^{\sigma, f}(N, E, c) \\
& =\frac{(|N|-1) !}{|N| !} f_{i}\left(\left\{i, i^{c}\right\}, E,\left(c_{i}, \sum_{j \in N \backslash\{i\}} c_{j}\right)\right)
\end{aligned}
$$




$$
\begin{aligned}
& +\frac{1}{|N| !} \sum_{j \in N \backslash\{i\}} \sum_{\bar{\sigma} \in \Pi(N \backslash\{j\})} s_{i}^{\bar{\sigma}, f}\left(N \backslash\{j\}, E_{-j}, c_{-j}\right) \\
= & \frac{1}{|N|} f_{i}\left(\left\{i, i^{c}\right\}, E,\left(c_{i}, \sum_{j \in N \backslash\{i\}} c_{j}\right)\right) \\
& +\frac{(|N|-1) !}{|N| !} \sum_{j \in N \backslash\{i\}} \frac{1}{(|N|-1) !} \sum_{\bar{\sigma} \in \Pi(N \backslash\{j\})} s_{i}^{\bar{\sigma}, f}\left(N \backslash\{j\}, E_{-j}, c_{-j}\right) \\
= & \frac{1}{|N|} f_{i}\left(\left\{i, i^{c}\right\}, E,\left(c_{i}, \sum_{j \in N \backslash\{i\}} c_{j}\right)\right) \\
& +\frac{1}{|N|} \sum_{j \in N \backslash\{i\}} R f_{i}\left(N \backslash\{j\}, E_{-j}, c_{-j}\right) .
\end{aligned}
$$

By means of Theorem 2.1 it readily follows (by induction) that for all $(N, E, c) \in \mathcal{C}$ it is true that $0 \leq R f(N, E, c) \leq c$ for each rule $f$. Moreover, it is readily seen that also $R f$ is anonymous. Hence, the random conjugate of a rule is indeed a rule itself.

In a problem with only two claimants, e.g., the run-to-the-bank rule (cf. O'Neill (1982)), the Aumann-Maschler rule (cf. Aumann and Maschler (1985)) and the adjusted proportional rule (cf. Curiel et al. (1988)) coincide. Hence, the random conjugates of these rules coincide too. Thomson (2003) refers to this basic or standard rule in two-claimant problems as concede-and-divide, abbreviated to CD and analogously we will refer to the random conjugates of the above three rules as the random concede-and-divide rule RCD. The related concede-and-divide principle for two-claimant problems is also known as the contested garment principle (Aumann and Maschler (1985)). It is based on the idea that each claimant concedes the amount of the estate that is not claimed by himself to the other claimant. This amount can be seen as a minimal right of a claimant. Subsequently, the amount left of the estate after giving both claimants their minimal rights is divided equally. A comprehensive discussion of the random concede-and-divide rule can be found in Quant et al. (2005). The next example illustrates the random concede-and-divide rule.

Example 2.1 Let $N=\{1,2,3\}, E=12$, and $c=(4,6,8)$. Consider the order $\sigma=(123)$. Then claimant 1 is the first claimant who leaves the group. Claimant 2 and 3 together have a total claim of 14 . The minimal right of claimant 1 equals 0 , and claimant 2 and 3 have together a minimal right of $E-c_{1}=8$. Claimant 1 receives $0+\frac{12-0-8}{2}=2$. Now claimant 2 is leaving and the remaining estate is $12-2=10$. The minimal rights of claimant 2 and 3 , respectively, are $10-8=2$ and $10-6=4$. Hence claimant 2 leaves with $2+\frac{10-2-4}{2}=4$, and claimant 3 receives $4+\frac{10-4-2}{2}=6$. This yields $s^{\sigma, C D}(N, E, c)=(2,4,6)$.

All vectors $s^{\sigma, C D}(N, E, c), \sigma \in \Pi(N)$ are given in the table below. 


\begin{tabular}{l|l}
$\sigma$ & $s^{\sigma, C D}(N, E, c)$ \\
\hline$(123)$ & $(2,4,6)$ \\
$(132)$ & $(2,4,6)$ \\
$(213)$ & $\left(2 \frac{1}{2}, 3,6 \frac{1}{2}\right)$ \\
$(231)$ & $\left(2 \frac{1}{2}, 3,6 \frac{1}{2}\right)$ \\
$(312)$ & $\left(2 \frac{1}{2}, 4 \frac{1}{2}, 5\right)$ \\
$(321)$ & $\left(2 \frac{1}{2}, 4 \frac{1}{2}, 5\right)$
\end{tabular}

And consequently

$$
R C D(N, E, c)=\frac{1}{6} \sum_{\sigma \in \Pi(N)} s^{\sigma, C D}(N, E, c)=\frac{1}{6}(14,23,35) .
$$

Note that the random concede-and-divide rule conceptually extends the concede-anddivide principle to arbitrary problems, but it differs from the run-to-the-bank rule, the adjusted proportional rule, and the Aumann-Maschler rule, which respectively give the allocations $\frac{1}{6}(16,22,34),\left(2 \frac{1}{2}, 3 \frac{3}{4}, 5 \frac{3}{4}\right)$, and $(2,4,6)$.

One could wonder whether the random concede-and-divide rule is the consensus value (introduced in Ju et al. (2004)) of the corresponding bankruptcy game, since both rules are based on an extension of the standard solution for the case when there are only two claimants/players present. However, the random concede-and-divide allocation of a bankruptcy problem generally does not equal the consensus value of the corresponding bankruptcy game. In fact the last approach does not determine a rule. ${ }^{2}$

The proportional rule is an example of a rule that does not satisfy the concedeand-divide principle in two-claimant problems. Let $(N, E, c) \in \mathcal{C}$. For all $i \in N$ the proportional rule (PROP) is defined by

$$
\operatorname{PROP}_{i}(N, E, c)=\frac{c_{i}}{\sum_{j \in N} c_{j}} \cdot E .
$$

With the help of the definition of $s^{\sigma, P R O P}(N, E, c)$, as given in Eq. 1, it is immediately seen that for all $\sigma \in \Pi(N)$, we have $s^{\sigma, P R O P}(N, E, c)=P R O P(N, E, c)$. Therefore, the random conjugate of the proportional rule coincides with the proportional rule itself, or stated differently the proportional rule is invariant under the operator that assigns to each two-claimant rule its random conjugate. In fact the proportional rule is the only rule $f$ for which $s^{\sigma, f}(N, E, c)=f(N, E, c)$ for all $(N, E, c) \in \mathcal{C}$ and all $\sigma \in \Pi(N)$. To prove this we use a characterization of the proportional rule based on a property which is called no advantageous merging or splitting: for all $(N, E, c) \in \mathcal{C}$ and all $T \subset N$ it is true that

$$
f_{t^{c}}\left(T \cup\left\{t^{c}\right\}, E, c^{T, t^{c}}\right)=\sum_{j \in N \backslash T} f_{j}(N, E, c),
$$

\footnotetext{
2 The following situation provides a counterexample: let $(N, E, c)=(\{1,2,3\}, 100,(60,60,1))$. The consensus value of the corresponding game equals $(48,48,4)$. For comparison, $R C D(N, E, c)=$ $\left(49 \frac{3}{4}, 49 \frac{3}{4}, \frac{1}{2}\right)$.
} 
where $t^{c}$, with minor abuse of notation, can be seen as the merging of all players in $N \backslash T$ and $c^{T, t^{c}} \in \mathbb{R}^{T \cup\left\{t^{c}\right\}}$ is the vector of claims such that $c_{j}^{T, t^{c}}=c_{j}$ if $j \in T$ and $c_{t^{c}}^{T, t^{c}}=\sum_{j \in N \backslash T} c_{j}$. In fact no advantageous merging or splitting states that merging a group of players to one player with added claims, in total yields the same payoff to these players. The proportional rule is the unique rule satisfying this property (cf. O’Neill (1982), Chun (1988), de Frutos (1999) and Ju et al. (2007)).

Theorem 2.2 Let $f$ be a rule and suppose that for all $(N, E, c) \in \mathcal{C}$ we have $f(N, E, c)=s^{\sigma, f}(N, E, c)$ for all $\sigma \in \Pi(N)$. Then $f$ is the proportional rule.

Proof It suffices to show that $f$ satisfies no advantageous merging or splitting.

Let $T \subset N$ and $\sigma \in \Pi(N)$ be such that for all $k \in\{1, \ldots,|T|\}, \sigma(k) \in T$. Hence, in the order $\sigma$ the claimants in $T$ are the first to leave. Define $\sigma^{*} \in \Pi\left(T \cup\left\{t^{c}\right\}\right)$ by $\sigma^{*}(k)=\sigma(k)$ for all $k \in\{1, \ldots,|T|\}$ and $\sigma^{*}(|T|+1)=t^{c}$. Now consider player $\sigma(1)$. Merging all the players in $N \backslash\{\sigma(1)\}$ yields a player with the same claim as the player that is the result of merging all the players in $T \cup\left\{t^{c}\right\} \backslash\{\sigma(1)\}$, and therefore,

$$
\begin{aligned}
& f_{\sigma(1)}\left(\left\{\sigma(1), \sigma(1)^{c}\right\}, E^{1},\left(c_{\sigma(1)}, \sum_{k=2}^{|N|} c_{\sigma(k)}\right)\right) \\
& =f_{\sigma^{*}(1)}\left(\left\{\sigma^{*}(1), \sigma^{*}(1)^{c}\right\}, E^{1},\left(c_{\sigma^{*}(1)}, \sum_{k=2}^{|T|+1} c_{\sigma^{*}(k)}^{T, t^{c}}\right)\right) .
\end{aligned}
$$

Hence $s_{\sigma(1)}^{\sigma, f}(N, E, c)=s_{\sigma^{*}(1)}^{\sigma^{*}, f}\left(T \cup\left\{t^{c}\right\}, E, c^{T, t^{c}}\right)$. Applying this argument $|T|$ times by using in each step that the estate and the claims of the two-claimant problems in both cases coincide, it follows that for all $k \in\{1, \ldots,|T|\}$ :

$$
s_{\sigma(k)}^{\sigma, f}(N, E, c)=s_{\sigma^{*}(k)}^{\sigma^{*}, f}\left(T \cup\left\{t^{c}\right\}, E, c^{T, t^{c}}\right) .
$$

Since $f$ is a rule, we know that:

$$
\sum_{k=1}^{|N|} s_{\sigma(k)}^{\sigma, f}(N, E, c)=E=\sum_{k=1}^{|T|+1} s_{\sigma^{*}(k)}^{\sigma^{*}, f}\left(T \cup\left\{t^{c}\right\}, E, c^{T, t^{c}}\right)
$$

This yields

$$
\begin{aligned}
f_{t^{c}}\left(T \cup\left\{t^{c}\right\}, E, c^{T, t^{c}}\right) & =s_{t^{c}}^{\sigma^{*}, f}\left(T \cup\left\{t^{c}\right\}, E, c^{T, t^{c}}\right) \\
& =s_{\sigma^{*}(|T|+1)}^{\sigma^{*}, f}\left(T \cup\left\{t^{c}\right\}, E, c^{T, t^{c}}\right) \\
& =\sum_{k=|T|+1}^{|N|} s_{\sigma(k)}^{\sigma, f}(N, E, c)
\end{aligned}
$$




$$
\begin{aligned}
& =\sum_{j \in N \backslash T} s_{j}^{\sigma, f}(N, E, c) \\
& =\sum_{j \in N \backslash T} f_{j}(N, E, c) .
\end{aligned}
$$

Here, the first and last equality follow from the assumption on $f$, that each order gives rise to the same allocation vector which is the outcome induced by $f$. The second equality follows from the definition of $\sigma^{*}$, the third equality from Eqs. 2 and 3, and finally, the fourth equality is implied by the definition of $\sigma$. So $f$ satisfies no advantageous merging or splitting, and therefore, $f$ is the proportional rule.

\section{Properties of random conjugates of rules}

In this section we analyze some general properties of the random conjugate of a rule. In particular we focus on the inheritance of special properties that are satisfied by the original rule in the two-claimant setting. For various rules characterizations are provided by a combination of properties by which this rule is uniquely determined. An overview of properties (and characterizations) of rules can be found in Thomson (2003). This section shows that many of these properties, but not all, are inherited by the random conjugate of a rule if the property is satisfied by the original rule in two-claimant problems.

For example, properties that are inherited by the random conjugate of a rule are homogeneity and resource monotonicity. Furthermore, claims monotonicity is preserved whenever a two-claimant rule satisfies both claims and resource monotonicity. ${ }^{3}$ The proofs are rather straightforward (by induction) and are left to the reader. In the following theorem we focus on the inheritance of the properties invariance under claims truncation, self-duality, and minimal rights first. These are properties that are frequently used in characterizations of several rules.

We first recall the definitions of these properties. Let $f$ be a rule. The rule $f$ is invariant under claims truncation if for all $(N, E, c) \in \mathcal{C}$, we have $f(N, E, c)=$ $f(N, E, \bar{c})$, where $\bar{c} \in \mathbb{R}^{N}$ is the truncated claim vector, i.e., $\overline{c_{i}}=\min \left\{E, c_{i}\right\}$ for all $i \in N$. The rule $f$ is self-dual if for all $(N, E, c) \in \mathcal{C}$, we have $f(N, E, c)=c-$ $f\left(N, \sum_{j \in N} c_{j}-E, c\right)$. The rule $f$ satisfies minimal rights first if for all $(N, E, c) \in$ $\mathcal{C}$, we have $f(N, E, c)=r(N, E, c)+f\left(N, E-\sum_{j \in N} r_{j}, c-r\right)$, where $r \in \mathbb{R}^{N}$ is the minimal right vector, i.e., $r_{i}(N, E, c)=\max \left\{0, E-\sum_{j \in N \backslash\{i\}} c_{j}\right\}$ for all $i \in N$. Note that the above three properties can also be defined for fixed claimants sizes only.

Theorem 3.1 Let $f$ be a rule.

(i) If $f$ satisfies invariance under claims truncation in two-claimant problems, then $R f$ satisfies invariance under claims truncation.

\footnotetext{
${ }^{3}$ Order preservation is preserved if a rule also satisfies resource monotonicity, claims monotonicity, and self-duality.
} 
(ii) If $f$ satisfies self-duality in two-claimant problems, then $R f$ satisfies self-duality.

(iii) If $f$ satisfies minimal rights first in two-claimant problems, then $R f$ satisfies minimal rights first.

Proof All proofs are given by an induction argument on the number of claimants present.

(i) Assume that $f$ satisfies invariance under claims truncation for two-claimant problems. Let $(N, E, c) \in \mathcal{C}$ and define $(N, E, \bar{c})$ by $\bar{c}_{i}=\min \left\{E, c_{i}\right\}$ for all $i \in N$. Let $|N|=2$. Since the random conjugate of a rule coincides with the original rule in the two-claimant case, it follows by the assumption that $R f(N, E, c)=R f(N, E, \bar{c})$ for $|N|=2$.

Let $k \in \mathbb{N}, k \geq 2$. Assume that for all problems with number of claimants less than or equal to $k$ invariance under claims truncation is satisfied. Let $N$ be such that $|N|=$ $k+1$. We will use the recursive formula of Theorem 2.1 to prove that $R f_{i}(N, E, c)=$ $R f_{i}(N, E, \bar{c})$ for all $i \in N$. Since $f$ is invariant under claims truncation for twoclaimant problems and because $\min \left\{E, \sum_{j \in N \backslash\{i\}} c_{j}\right\}=\min \left\{E, \sum_{j \in N \backslash\{i\}} \bar{c}_{j}\right\}$ it is true that (with notations as in Theorem 2.1)

$$
\begin{aligned}
R f_{i}(N, E, c)= & \frac{1}{|N|} f_{i}\left(\left\{i, i^{c}\right\}, E,\left(c_{i}, \sum_{j \in N \backslash\{i\}} c_{j}\right)\right) \\
& +\frac{1}{|N|} \sum_{j \in N \backslash\{i\}} R f_{i}\left(N \backslash\{j\}, E_{-j}, c_{-j}\right) \\
= & \frac{1}{|N|} f_{i}\left(\left\{i, i^{c}\right\}, E,\left(\bar{c}_{i}, \min \left\{E, \sum_{j \in N \backslash\{i\}} c_{j}\right\}\right)\right) \\
& +\frac{1}{|N|} \sum_{j \in N \backslash\{i\}} R f_{i}\left(N \backslash\{j\}, E_{-j}, c_{-j}\right) \\
= & \left.\frac{1}{|N|} f_{i}\left(\left\{i, i^{c}\right\}, E,\left(\bar{c}_{i}, \min _{E^{\prime}} \sum_{j \in N \backslash\{i\}} \bar{c}_{j}\right\}\right)\right) \\
& +\frac{1}{|N|} \sum_{j \in N \backslash\{i\}} R f_{i}\left(N \backslash\{j\}, E_{-j}, c_{-j}\right) \\
= & \frac{1}{|N|} f_{i}\left(\left\{i, i^{c}\right\}, E,\left(\bar{c}_{i}, \sum_{j \in N \backslash\{i\}} \bar{c}_{j}\right)\right) \\
& +\frac{1}{|N|} \sum_{j \in N \backslash\{i\}} R f_{i}\left(N \backslash\{j\}, E_{-j}, c_{-j}\right)
\end{aligned}
$$

Let $j \in N \backslash\{i\}$ and let $\tilde{c} \in \mathbb{R}^{N \backslash\{j\}}$ be defined as $\tilde{c}_{k}=\min \left\{E_{-j}, c_{k}\right\}$ for all $k \in N \backslash\{j\}$. Then also $\tilde{c}_{k}=\min \left\{E_{-j}, \bar{c}_{k}\right\}$. Using the induction hypothesis twice we find that

$$
R f\left(N \backslash\{j\}, E_{-j}, c_{-j}\right)=R f\left(N \backslash\{j\}, E_{-j}, \tilde{c}_{-j}\right),
$$


and

$$
R f\left(N \backslash\{j\}, E_{-j}, \bar{c}_{-j}\right)=R f\left(N \backslash\{j\}, E_{-j}, \tilde{c}_{-j}\right) .
$$

Using the above observations, formula (4) can be rewritten as

$$
\begin{aligned}
R f_{i}(N, E, c)= & \frac{1}{|N|} f_{i}\left(\left\{i, i^{c}\right\}, E,\left(\bar{c}_{i}, \sum_{j \in N \backslash\{i\}} \bar{c}_{j}\right)\right) \\
& +\frac{1}{|N|} \sum_{j \in N \backslash\{i\}} R f_{i}\left(N \backslash\{j\}, E_{-j}, \bar{c}_{-j}\right) \\
= & R f_{i}(N, E, \bar{c}) .
\end{aligned}
$$

We may conclude that $R f$ satisfies invariance under claims truncation for arbitrary population sizes.

(ii) Assume that $f$ satisfies self-duality for two-claimant problems. We first note that to prove self-duality of $R f$, it is sufficient to prove that for all $\sigma \in \Pi(N)$ and all $(N, E, c)$ we have that

$$
s^{\sigma, f}(N, E, c)=c-s^{\sigma, f}\left(N, \sum_{j \in N} c_{j}-E, c\right) .
$$

That (5) is valid if $|N|=2$ is obvious, because $f$ is self-dual in two-claimant problems and $f(N, E, c)=R f(N, E, c)$ for all problems $(N, E, c)$ with $|N|=2$.

Let $k \in \mathbb{N}, k \geq 2$. Assume that for all problems $(N, E, c)$ such that $|N| \leq k$ and all $\sigma \in \Pi(N)$, formula (5) is satisfied. Take $(N, E, c) \in \mathcal{C}$, such that $|N|=k+1$ and let $\sigma \in \Pi(N)$. Denote $\sum_{j \in N} c_{j}$ by $C$. We first prove that

$$
s_{\sigma(1)}^{\sigma, f}(N, E, c)+s_{\sigma(1)}^{\sigma, f}(N, C-E, c)=c_{\sigma(1)} .
$$

Since $\sigma(1)$ is the first claimant who leaves the group, we have that

$$
s_{\sigma(1)}^{\sigma, f}(N, E, c)=f_{\sigma(1)}\left(\left\{\sigma(1), \sigma(1)^{c}\right\}, E,\left(c_{\sigma(1)}, C-c_{\sigma(1)}\right)\right),
$$

and

$$
s_{\sigma(1)}^{\sigma, f}(N, C-E, c)=f_{\sigma(1)}\left(\left\{\sigma(1), \sigma(1)^{c}\right\}, C-E,\left(c_{\sigma(1)}, C-c_{\sigma(1)}\right)\right) .
$$

Now Eq. 6 immediately follows by adding Eqs. 7 and 8 and using the self-duality of $f$ in a problem with only two claimants.

Let $E_{-\sigma(1)}$ denote the amount that is left of $E$, if $\sigma(1)$ has first left the group in the problem $(N, E, c)$ and similarly $(C-E)_{-\sigma(1)}$ is the amount that is left of $E$, if $\sigma(1)$ has first left the group in the problem $(N, C-E, c)$. From Eq. 6 it follows that

$$
E_{-\sigma(1)}+(C-E)_{-\sigma(1)}=C-c_{\sigma(1)} .
$$


Let $\bar{\sigma} \in \Pi(N \backslash\{\sigma(1)\})$ be the order of all other claimants that is induced by $\sigma$. Let $i \in N \backslash\{\sigma(1)\}$. Then

$$
\begin{aligned}
s_{i}^{\sigma, f}(N, E, c) & =s_{i}^{\bar{\sigma}, f}\left(N \backslash\{\sigma(1)\}, E_{-\sigma(1)}, c_{-\sigma(1)}\right) \\
& =c_{i}-s_{i}^{\bar{\sigma}, f}\left(N \backslash\{\sigma(1)\}, C-c_{\sigma(1)}-E_{-\sigma(1)}, c_{-\sigma(1)}\right) \\
& \stackrel{(9)}{=} c_{i}-s_{i}^{\bar{\sigma}, f}\left(N \backslash\{\sigma(1)\},(C-E)_{-\sigma(1)}, c_{-\sigma(1)}\right) \\
& =c_{i}-s_{i}^{\sigma, f}(N, C-E, c) .
\end{aligned}
$$

The second equality follows from the induction hypothesis. This completes the induction argument, $R f$ satisfies self-duality.

(iii) Assume that $f$ satisfies minimal rights first for two-claimant problems. Note that if for all $(N, E, c)$ and all $\sigma \in \Pi(N)$ we have that

$$
s^{\sigma, f}(N, E, c)=r(N, E, c)+s^{\sigma, f}\left(N, E-\sum_{j \in N} r_{j}(N, E, c), c-r(N, E, c)\right),
$$

then $R f$ satisfies minimal rights first. For $|N|=2$, Eq. 10 is true, because $f$ satisfies minimal rights first for $|N|=2$.

Let $k \in \mathbb{N}, k \geq 2$. Assume that for all problems $(N, E, c)$ such that $|N| \leq k$ and for all $\sigma \in \Pi(N)$, formula (10) is satisfied. Let $(N, E, c) \in \mathcal{C}$ be such that $|N|=k+1$ and let $\sigma \in \Pi(N)$. For ease of notation we define $R(B)=\sum_{j \in N} r_{j}(B)$ for a problem $B \in \mathcal{C}$. We first prove that

$$
s_{\sigma(1)}^{\sigma, f}(N, E, c)=r_{\sigma(1)}(N, E, c)+s_{\sigma(1)}^{\sigma, f}(N, E-R(N, E, c), c-r(N, E, c)) .
$$

For ease of notation we define the following problems:

$$
\begin{aligned}
B_{1}:= & (N, E, c), \\
B_{2}:= & \left(\left\{\sigma(1), \sigma(1)^{c}\right\}, E,\left(c_{\sigma(1)}, C-c_{\sigma(1)}\right)\right), \\
B_{3}:= & \left(\left\{\sigma(1), \sigma(1)^{c}\right\}, E-R\left(B_{2}\right),\left(c_{\sigma(1)}-r_{\sigma(1)}\left(B_{2}\right), C-c_{\sigma(1)}-r_{\sigma(1)^{c}}\left(B_{2}\right)\right)\right) \\
B_{4}:= & \left(N, E-R\left(B_{1}\right), c-r\left(B_{1}\right)\right) \\
B_{5}:= & \left(\left\{\sigma(1), \sigma(1)^{c}\right\}, E-R\left(B_{1}\right),\left(c_{\sigma(1)}-r_{\sigma(1)}\left(B_{1}\right),\right.\right. \\
& \left.\left.C-\left(c_{\sigma(1)}-r_{\sigma(1)}\left(B_{1}\right)\right)-R\left(B_{1}\right)\right)\right)
\end{aligned}
$$

As $f$ satisfies minimal rights first in the case $|N|=2$, we can deduce that

$$
\begin{aligned}
s_{\sigma(1)}^{\sigma, f}(N, E, c) & =s_{\sigma(1)}^{\sigma, f}\left(B_{1}\right) \\
& =f_{\sigma(1)}\left(B_{2}\right) \\
& =r_{\sigma(1)}\left(B_{2}\right)+f_{\sigma(1)}\left(B_{3}\right) \\
& =r_{\sigma(1)}\left(B_{1}\right)+f_{\sigma(1)}\left(B_{3}\right),
\end{aligned}
$$


where the last equality follows from

$$
r_{\sigma(1)}\left(B_{1}\right)=\max \left\{0, E-\sum_{j \in N \backslash\{\sigma(1)\}} c_{j}\right\}=\max \left\{0, E-\left(C-c_{\sigma(1)}\right)\right\}=r_{\sigma(1)}\left(B_{2}\right) .
$$

On the other hand

$$
r_{\sigma(1)}\left(B_{1}\right)+s_{\sigma(1)}^{\sigma, f}\left(B_{4}\right)=r_{\sigma(1)}\left(B_{1}\right)+f_{\sigma(1)}\left(B_{5}\right)
$$

So for (11) to be true we need to prove that $f_{\sigma(1)}\left(B_{3}\right)=f_{\sigma(1)}\left(B_{5}\right)$. We distinguish between two cases.

Case 1: $r_{i}\left(B_{1}\right)=0$ for all $i \in N \backslash\{\sigma(1)\}$.

This implies that $R\left(B_{1}\right)=r_{\sigma(1)}\left(B_{1}\right)$ and $r_{\sigma(1)}\left(B_{5}\right)=0$. Hence, using minimal rights first for two-claimant problems

$$
\begin{aligned}
& f_{\sigma(1)}\left(B_{5}\right) \\
& \quad=f_{\sigma(1)}\left(\left\{\sigma(1), \sigma(1)^{c}\right\}, E-r_{\sigma(1)}\left(B_{1}\right)-r_{\sigma(1)^{c}}\left(B_{5}\right),\left(c_{\sigma(1)}-r_{\sigma(1)}\left(B_{1}\right),\right.\right. \\
& \left.\left.\quad C-c_{\sigma(1)}-r_{\sigma(1)^{c}}\left(B_{5}\right)\right)\right) \\
& \stackrel{(12)}{=} f_{\sigma(1)}\left(\left\{\sigma(1), \sigma(1)^{c}\right\}, E-r_{\sigma(1)}\left(B_{2}\right)-r_{\sigma(1)^{c}}\left(B_{5}\right),\left(c_{\sigma(1)}-r_{\sigma(1)}\left(B_{2}\right),\right.\right. \\
& \left.\left.C-c_{\sigma(1)}-r_{\sigma(1)^{c}}\left(B_{5}\right)\right)\right) .
\end{aligned}
$$

Since

$$
\begin{aligned}
r_{\sigma(1)^{c}}\left(B_{5}\right) & =\max \left\{0, E-r_{\sigma(1)}\left(B_{1}\right)-\left(c_{\sigma(1)}-r_{\sigma(1)}\left(B_{1}\right)\right)\right\} \\
& =\max \left\{0, E-c_{\sigma(1)}\right\} \\
& =r_{\sigma(1)^{c}}\left(B_{2}\right),
\end{aligned}
$$

it follows that $f_{\sigma(1)}\left(B_{3}\right)=f_{\sigma(1)}\left(B_{5}\right)$.

Case 2: there is $i \in N \backslash\{\sigma(1)\}$ such that $r_{i}\left(B_{1}\right)>0$.

It follows that $c_{\sigma(1)}<E$ and also

$c_{\sigma(1)}-r_{\sigma(1)}\left(B_{1}\right)<E-r_{\sigma(1)}\left(B_{1}\right)$. Furthermore

$$
r_{i}\left(B_{1}\right)=E-\sum_{j \in N \backslash\{i\}} c_{j} \leq E-\sum_{j \in N \backslash\{\sigma(1), i\}} r_{j}\left(B_{1}\right)-c_{\sigma(1)}
$$


implying that $c_{\sigma(1)}-r_{\sigma(1)}\left(B_{1}\right) \leq E-R\left(B_{1}\right)$. From this we can derive that

$$
\begin{aligned}
r_{\sigma(1)^{c}}\left(B_{5}\right) & =\max \left\{0, E-R\left(B_{1}\right)-\left(c_{\sigma(1)}-r_{\sigma(1)}\left(B_{1}\right)\right)\right\} \\
& =E-R\left(B_{1}\right)-\left(c_{\sigma(1)}-r_{\sigma(1)}\left(B_{1}\right)\right), \\
r_{\sigma(1)^{c}}\left(B_{2}\right) & =\max \left\{0, E-c_{\sigma(1)}\right\}=E-c_{\sigma(1)} .
\end{aligned}
$$

and, hence,

$$
\begin{aligned}
E-R\left(B_{1}\right)-r_{\sigma(1)^{c}}\left(B_{5}\right) & =E-R\left(B_{1}\right)-\left(E-R\left(B_{1}\right)-\left(c_{\sigma(1)}-r_{\sigma(1)}\left(B_{1}\right)\right)\right) \\
& =c_{\sigma(1)}-r_{\sigma(1)}\left(B_{1}\right) \\
& =E-r_{\sigma(1)}\left(B_{1}\right)-\left(E-c_{\sigma(1)}\right) \\
& =E-r_{\sigma(1)}\left(B_{2}\right)-r_{\sigma(1)^{c}}\left(B_{2}\right) \\
& =E-R\left(B_{2}\right) .
\end{aligned}
$$

Since $\mathrm{f}$ satisfies minimal rights first in two-claimant problems (and hence especially in problem $\left.B_{5}\right)$, the fact that $r_{\sigma(1)}\left(B_{5}\right)=0$ and using equality (12) and the above results, we find that $f_{\sigma(1)}\left(B_{5}\right)$ equals

$$
\begin{aligned}
& f_{\sigma(1)}\left(\left\{\sigma(1), \sigma(1)^{c}\right\}, E-R\left(B_{2}\right),\left(c_{\sigma(1)}-r_{\sigma(1)}\left(B_{2}\right), C-\left(c_{\sigma(1)}-r_{\sigma(1)}\left(B_{2}\right)\right)\right.\right. \\
& \left.\left.\quad-R\left(B_{1}\right)-r_{\sigma(1)^{c}}\left(B_{5}\right)\right)\right) .
\end{aligned}
$$

By using (12) and (13), we can derive that

$$
\begin{aligned}
C & -\left(c_{\sigma(1)}-r_{\sigma(1)}\left(B_{2}\right)\right)-R\left(B_{1}\right)-r_{\sigma(1)^{c}}\left(B_{5}\right) \\
& =C-\left(c_{\sigma(1)}-r_{\sigma(1)}\left(B_{2}\right)\right)-R\left(B_{1}\right)-\left(E-R\left(B_{1}\right)-\left(c_{\sigma(1)}-r_{\sigma(1)}\left(B_{1}\right)\right)\right) \\
& =C-E \\
& =C-c_{\sigma(1)}-\left(E-c_{\sigma(1)}\right)=C-c_{\sigma(1)}-r_{\sigma(1)^{c}}\left(B_{2}\right) .
\end{aligned}
$$

and hence $f_{\sigma(1)}\left(B_{5}\right)=f_{\sigma(1)}\left(B_{3}\right)$, which establishes (11).

Now Eq. 11 is proved, it remains to show that for each $i \in N \backslash\{\sigma(1)\}$

$$
s_{i}^{\sigma, f}(N, E, c)=r_{i}(N, E, c)+s_{i}^{\sigma, f}(N, E-R(N, E, c), c-r(N, E, c))
$$

This boils down to proving the following claim.

Claim: $\quad s_{i}^{\sigma, f}(N, E, c)=r_{i}\left(B_{1}\right)+s_{i}^{\sigma, f}\left(B_{4}\right), \quad$ for all $i \in N \backslash \sigma(1)$.

To do so, we also define, additional to $B_{1}, \ldots, B_{5}$, the problems

$$
\begin{aligned}
& B_{6}:=\left(N \backslash\{\sigma(1)\}, E_{-\sigma(1)}, c_{-\sigma(1)}\right), \\
& B_{7}:=\left(N \backslash\{\sigma(1)\}, E_{-\sigma(1)}-R\left(B_{1}\right)+r_{\sigma(1)}\left(B_{1}\right),\left(c-r\left(B_{1}\right)\right)_{-\sigma(1)}\right), \\
& B_{8}:=\left(N \backslash\{\sigma(1)\}, E_{-\sigma(1)}-R\left(B_{1}\right)+r_{\sigma(1)}\left(B_{1}\right)-R\left(B_{7}\right),(c-r)_{-\sigma(1)}-r\left(B_{7}\right)\right) .
\end{aligned}
$$


From the definition of $E_{-\sigma(1)}$ and $\left(E-R\left(B_{1}\right)\right)_{-\sigma(1)}$, it can be concluded that

$$
\begin{aligned}
E_{-\sigma(1)}-R\left(B_{1}\right) & =E-s_{\sigma(1)}^{\sigma, f}\left(B_{1}\right)-R\left(B_{1}\right) \\
& \stackrel{(11)}{=} E-r_{\sigma(1)}\left(B_{1}\right)-s_{\sigma(1)}^{\sigma, f}\left(B_{4}\right)-R\left(B_{1}\right) \\
& =\left(E-R\left(B_{1}\right)\right)_{-\sigma(1)}-r_{\sigma(1)}\left(B_{1}\right)
\end{aligned}
$$

Let $\bar{\sigma} \in \Pi(N \backslash \sigma(1))$ be the order of all other claimants that is induced by $\sigma$.

Now let $i \in N \backslash\{\sigma(1)\}$. Using the induction hypothesis we find

$$
\begin{aligned}
s_{i}^{\sigma, f}\left(B_{1}\right) & =s_{i}^{\bar{\sigma}, f}\left(B_{6}\right) \\
& =r_{i}\left(B_{6}\right)+s_{i}^{\bar{\sigma}, f}\left(N \backslash\{\sigma(1)\}, E_{-\sigma(1)}-R\left(B_{6}\right), c_{-\sigma(1)}-r\left(B_{6}\right)\right) .
\end{aligned}
$$

On the other hand

$r_{i}\left(B_{1}\right)+s_{i}^{\sigma, f}\left(B_{4}\right)=r_{i}\left(B_{1}\right)+s_{i}^{\bar{\sigma}, f}\left(N \backslash\{\sigma(1)\},\left(E-R\left(B_{1}\right)\right)_{-\sigma(1)},\left(c-r\left(B_{1}\right)_{-\sigma(1)}\right)\right.$,

and hence, by (15)

$$
r_{i}\left(B_{1}\right)+s_{i}^{\sigma, f}\left(B_{4}\right)=r_{i}\left(B_{1}\right)+s_{i}^{\bar{\sigma}, f}\left(B_{7}\right) .
$$

Since $|N \backslash\{\sigma(1)\}|=k$, we can apply the induction hypothesis to the problem $B_{7}$. For this we first calculate the minimal right $r_{i}\left(B_{7}\right)$ :

$$
\begin{aligned}
r_{i}\left(B_{7}\right) & =\max \left\{E_{-\sigma(1)}-R\left(B_{1}\right)+r_{\sigma(1)}\left(B_{1}\right)-\sum_{j \in N \backslash\{i, \sigma(1)\}}\left(c_{j}-r_{j}\left(B_{1}\right)\right), 0\right\} \\
& =\max \left\{E_{-\sigma(1)}-r_{i}\left(B_{1}\right)-\sum_{j \in N \backslash\{i, \sigma(1)\}} c_{j}, 0\right\} \\
& =\max \left\{E_{-\sigma(1)}-\sum_{j \in N \backslash\{i, \sigma(1)\}} c_{j}, r_{i}\left(B_{1}\right)\right\}-r_{i}\left(B_{1}\right) \\
& =\max \left\{r_{i}\left(B_{6}\right), r_{i}\left(B_{1}\right)\right\}-r_{i}\left(B_{1}\right) \\
& =r_{i}\left(B_{6}\right)-r_{i}\left(B_{1}\right) .
\end{aligned}
$$

The last equality follows, since $E_{-\sigma(1)} \geq E-c_{\sigma(1)}$ and hence $r_{i}\left(B_{6}\right) \geq r_{i}\left(B_{1}\right)$. We can deduce that

$$
\begin{aligned}
r_{i}\left(B_{1}\right)+s_{i}^{\sigma, f}\left(B_{4}\right) & =r_{i}\left(B_{1}\right)+r_{i}\left(B_{7}\right)+s_{i}^{\bar{\sigma}, f}\left(B_{8}\right) \\
& \stackrel{(17)}{=} r_{i}\left(B_{6}\right)+s_{i}^{\bar{\sigma}, f}\left(N \backslash\{\sigma(1)\}, E_{-\sigma(1)}-R\left(B_{6}\right), c_{-\sigma(1)}-r\left(B_{6}\right)\right) .
\end{aligned}
$$


According to Eq. 16 this indeed shows that $s_{i}^{\sigma, f}\left(B_{1}\right)=r_{i}\left(B_{1}\right)+s_{i}^{\sigma, f}\left(B_{4}\right)$ and our claim (cf. (14)) is proven.

This completes the proof of minimal rights first of $R f$.

Not all properties are inherited by the random conjugate of a rule, which is shown in the following example. This example illustrates that composition down is not necessarily inherited. A rule $f$ satisfies composition down if for $(N, E, c) \in \mathcal{C}$ and each $E^{\prime}<E$ we have

$$
f\left(N, E^{\prime}, c\right)=f\left(N, E^{\prime}, f(N, E, c)\right)
$$

The example uses the constrained equal awards rule. The constrained equal awards rule (abbreviated to CEA) is a rule that satisfies composition down. The allocation prescribed by the CEA rule for a problem $(N, E, c) \in \mathcal{C}$ is given by

$$
\mathrm{CEA}_{i}(N, E, c)=\min \left\{\alpha, c_{i}\right\},
$$

for all $i \in N$, where $\alpha$ is chosen such that $\sum_{i \in N} \operatorname{CEA}_{i}(N, E, c)=E$.

Example 3.1 Consider the problem $(N, E, c)$ with $N=\{1,2,3\}, E=100$, and $c=$ $(70,50,30)$ and let $E^{\prime}=90$. We first calculate $\operatorname{RCEA}(N, E, c)$ and $\operatorname{RCEA}\left(N, E^{\prime}, c\right)$. The following table contains the vectors $s^{\sigma, C E A}(N, E, c)$ and $s^{\sigma, C E A}\left(N, E^{\prime}, c\right)$, for all $\sigma \in \Pi(N)$.

\begin{tabular}{l|l|l}
$\sigma$ & $s^{\sigma, C E A}(N, E, c)$ & $s^{\sigma, C E A}\left(N, E^{\prime}, c\right)$ \\
\hline$(123)$ & $(50,25,25)$ & $\left(45,22 \frac{1}{2}, 22 \frac{1}{2}\right)$ \\
$(132)$ & $(50,25,25)$ & $\left(45,22 \frac{1}{2}, 22 \frac{1}{2}\right)$ \\
$(213)$ & $(25,50,25)$ & $\left(22 \frac{1}{2}, 45,22 \frac{1}{2}\right)$ \\
$(231)$ & $(25,50,25)$ & $\left(22 \frac{1}{2}, 45,22 \frac{1}{2}\right)$ \\
$(312)$ & $(35,35,30)$ & $(30,30,30)$ \\
$(321)$ & $(35,35,30)$ & $(30,30,30)$
\end{tabular}

Consequently

$\operatorname{RCEA}(N, E, c)=\frac{1}{6}(220,220,160)$ and $\operatorname{RCEA}\left(N, E^{\prime}, c\right)=\frac{1}{6}(195,195,150)$.

Next we calculate $\operatorname{RCEA}\left(N, E^{\prime}, \operatorname{RCEA}(N, E, c)\right)$. The corresponding vectors $s^{\sigma, C E A}\left(N, E^{\prime}, \operatorname{RCEA}(N, E, c)\right)$ are given below. 


\begin{tabular}{l|l}
$\sigma$ & $s^{\sigma, C E A}\left(N, E^{\prime}, \operatorname{RCEA}(N, E, c)\right)$ \\
\hline$(123)$ & $\frac{1}{6}(220,160,160)$ \\
$(132)$ & $\frac{1}{6}(220,160,160)$ \\
$(213)$ & $\frac{1}{6}(160,220,160)$ \\
$(231)$ & $\frac{1}{6}(160,220,160)$ \\
$(312)$ & $\frac{1}{6}(190,190,160)$ \\
$(321)$ & $\frac{1}{6}(190,190,160)$
\end{tabular}

As a result

$$
\operatorname{RCEA}\left(N, E^{\prime}, \operatorname{RCEA}(N, E, c)\right)=\frac{1}{6}(190,190,160),
$$

which does not equal $\operatorname{RCEA}\left(N, E^{\prime}, c\right)$. We conclude that RCEA does not satisfy composition down, although CEA does.

As is proved in Dagan (1996), the CEA rule is the unique rule that satisfies equal treatment of equals, invariance under claims truncation, and composition up. The random conjugate RCEA also satisfies equal treatment of equals and invariance under claims truncation, since both properties are inherited by the random conjugate of a rule. As the above example shows RCEA differs from CEA, hence RCEA does not satisfy composition up. This means that composition up is not necessarily inherited by the random conjugate of a rule.

Acknowledgements The authors thank William Thomson for helpful comments.

Open Access This article is distributed under the terms of the Creative Commons Attribution Noncommercial License which permits any noncommercial use, distribution, and reproduction in any medium, provided the original author(s) and source are credited.

\section{References}

Aumann R, Maschler M (1985) Game theoretic analysis of a bankruptcy problem from the Talmud. J Econ Theory 36:195-213

Chun Y (1988) The proportional solutions for rights problems. Math Soc Sci 15:231-246

Curiel I, Maschler M, Tijs S (1988) Bankruptcy games. Zeitschrift für Operations Research 31:143-159

Dagan N (1996) New characterizations of old bankruptcy rules. Soc Choice Welf 13:51-59

Dagan N, Volij O (1997) Bilateral comparisons and consistent fair division rules in the context of bankruptcy problems. Int J Game Theory 26:11-25

de Frutos M (1999) Coalitional manipulation in a bankruptcy problem. Rev Econ Des 4:255-272

Dominguez D, Thomson W (2006) A new solution to the problem of adjudicating conflicting claims. Econ Theory 28:283-307

Hokari T, Thomson W (2008) On properties of division rules lifted by bilateral consistency. J Math Econ 44:1057-1071

Ju Y, Borm P, Ruys P (2004) The consensus value: a new solution concept for cooperative games. CentER DP 2004-50, Tilburg University, Tilburg, The Netherlands

Ju B-G, Miyagawa E, Sakai T (2007) Non-manipulable division rules in claim problems and generalizations. J Econ Theory 132:1-26

Moulin H (2000) Priority rules and other assymetric rationing methods. Econometrica 68:643-684

O’Neill B (1982) A problem of rights arbitration from the Talmud. Math Soc Sci 2:345-371 
Quant M, Borm P, Maaten R (2005) A concede-and-divide rule for bankruptcy problems. CentER DP 2005-20, Tilburg University, Tilburg, The Netherlands

Thomson W (2003) Axiomatic and game theoretic analysis of bankruptcy and taxation problems: a survey. Math Soc Sci 45:249-297

Thomson W (2007) On the existence of consistent rules to adjucidate conflicting claims: a constructive geometric approach. Rev Econ Des 11:225-251

Thomson W (2008) The two-agent claims-truncated proportional rule has no consistent extension: a constructive proof. Econ Lett 98:59-65

Thomson W, Yeh C (2008) Operators for the adjudication of conflicting claims. J Econ Theory 143:177-198 